\title{
Assessment of Vancomycin MIC Creep Phenomenon in Methicillin-Resistant Staphylococcus aureus isolates in a Tertiary Care Hospital of Lahore
}

\author{
Faiqa Arshad', Sidrah Saleem², \\ Shah Jahan ${ }^{3}$, Romeeza Tahir ${ }^{4}$
}

\section{ABSTRACT}

Objective: To assess vancomycin MIC creep phenomenon in methicillin-resistant Staphylococcus aureus isolated from clinical specimens.

Methods: This descriptive study was conducted in Microbiology department of University of Health Sciences, Lahore from January 2016- December 2019. In this study, vancomycin MICs were revealed by E test method for clinical MRSA strains. For the final evaluation, a single isolate from each patient was taken. The reported vancomycin MICs results were used and the values were not rounded up to the next upward value. For every study year, MIC50, MIC90, median and geometrical mean MIC, percentages of susceptible and resistant strains were calculated.

Results: A total of 352 MRSA strains were isolated out of 2704 staphylococcal isolates. Our study showed elevated vancomycin MIC among MRSA isolates. The majority of isolates showed MIC values $\geq 1.5 \mu \mathrm{g} / \mathrm{ml}$. MIC50, MIC 90 was constant throughout four years period. However, geometric mean MIC increased gradually during the study period. The MIC greater than base year median was overall $17.3 \%$. A complete shift can be observed between MIC "1.0" and "2.0" the percent of cases with MIC " 1.0 " decreased and with MIC "2.0" increased over time crossing each other in 2017.

Conclusion: Vancomycin MIC creep was identified in clinical isolates of MRSA, during four years of study period. Even though there is an absence of VISA and VRSA strains; this significant increase in vancomycin MIC trend is indeed worrying for the clinicians about the threat of potential failure of treatment in MRSA infections.

KEYWORDS: Methicillin-resistant Staphylococcus aureus (MRSA); Vancomycin intermediate Staphylococcus aureus (VISA); Vancomycin-resistant Staphylococcus aureus (VRSA); Minimum inhibitory concentration (MIC).

doi: https://doi.org/10.12669/pjms.36.7.3273

How to cite this:

Arshad F, Saleem S, Jahan S, Tahir R. Assessment of Vancomycin MIC Creep Phenomenon in Methicillin-Resistant Staphylococcus aureus isolates in a Tertiary Care Hospital of Lahore. Pak J Med Sci. 2020;36(7):1505-1510. doi: https://doi.org/10.12669/pjms.36.7.3273

This is an Open Access article distributed under the terms of the Creative Commons Attribution License (http://creativecommons.org/licenses/by/3.0), which permits unrestricted use, distribution, and reproduction in any medium, provided the original work is properly cited.

Correspondence:

Dr. Faiqa Arshad, Ph.D. Scholar, Department of Microbiology,

University of Health Sciences,

Lahore, Pakistan.

Postal Address: 631-A Karim Block,

Allama lqbal Town,

Lahore, Pakistan.

Email: microbiologyuhs123@gmail.com

* Received for Publication:

* Revision Received:

* Revision Accepted: *
July 13,2020

September 7, 2020

September 13, 2020

\section{INTRODUCTION}

MRSA is a superbug and is a serious human health issue that is considered as one of the foremost causes of healthcare and community acquired infections. ${ }^{1}$ MRSA is unaffected by methicillin and many lactam antibiotics, such as oxacillin, cefoxitin, cephalosporins and carbapenems. ${ }^{2}$ The strains of MRSA have been now endemic in a large number of hospitals throughout the world and predominantly affect patients, who have 
undergone major surgical procedures and those who are in intensive care units. ${ }^{3}$

Vancomycin has remained a mainstay of treatment for serious MRSA infections for many years. ${ }^{4}$ However, an increase in vancomycin MIC values in MRSA isolates is a serious concern because it has been indicated in many studies that the patients with high vancomycin MIC values even within the sensitive limit are ending with treatment failure. ${ }^{5}$ This rise in vancomycin MIC standards over the period is called "MIC creep". The term "creep" may be classified as a "gradual and unnoticed movement or shift." Thus, vancomycin MIC creep should be known as a steady rise in the central disposition of the vancomycin MIC levels. It results from the long term and excessive use of vancomycin under suboptimal doses. ${ }^{6}$

Creep phenomenon is globally reported to result in therapeutic failure, increase morbidity and increase relapse rate, slow clinical response and higher relapse rate. ${ }^{7}$ Evidently, creep is a regional problem consequently, local evaluation of susceptibility profiles is important for the clinical management of local MRSA infection. ${ }^{8}$ Now, there is a need to assess the existence of creep trend and warn the clinicians of these disastrous strains. ${ }^{9}$ Suitable analytical patterns for evaluation of the correlation between vancomycin MICs and scientific consequence of MRSA infections need to be investigated. ${ }^{10}$ Therefore, there is a dire need to identify the trend of vancomycin MICs in our local area.

The present research was designed to assess MIC creep for clinical isolates of MRSA against vancomycin over a 4-year period in a tertiary care institute, Lahore.

\section{METHODS}

The study was carried out after Ethics Committee/IRB approval with number UHS/ Education/126-18/3731, dated 23-11-2018. In this study, MRSA strains were obtained from different clinical specimens of hospitalized subjects with MRSA infection recognized from medical history and clinical microbiology laboratory, from 2016-2019 at Lahore General Hospital Lahore. The samples included blood, pus, wound swab, respiratory tract (tracheal aspirate and bronchoalveolar lavage) CSF, synovial fluid, urine and sputum. Only one sample per patient was incorporated into the study. Only the first isolate was tested in case there were more than one sample from the same patient.
Over-all 352 isolates were recovered from 2704 S.aureus isolates from various clinical samples of in-door patients. The specimens processed in the microbiology lab of the University of Health Sciences, Lahore were inoculated on blood agar plates and incubation was done at $35-37^{\circ} \mathrm{C}$ aerobically for 24hours. Following standard microbiological techniques; primary detection of S. aureus was carried out by observing the colony morphology on blood agar plates, Gram staining and catalase. Biochemical tests like coagulase and DNase were done for organism confirmation. The phenotypic screening for methicillin resistance was determined by the modified Kirby-Bauer disc diffusion method using $30 \mu \mathrm{g}$ cefoxitin disc (Oxoid) according to CLSI guiding principles. ${ }^{11}$ A bacterial suspension was adjusted for each strain, according to 0.5 McFarland turbidity standards and inoculation was done on Mueller Hinton agar (MHA). Zone of inhibition was determined after overnight incubation at $35^{\circ} \mathrm{C}$. The results were interpreted according to CLSI standards 2019. MRSA ATCC 33591 and methicillin-susceptible S. aureus (MSSA) ATCC 25923 were used as Controls in parallel while performing all phenotypic and genotypic test runs. ${ }^{11}$

MICs of vancomycin were detected by E-strips. The inoculum was prepared according to $0.5 \mathrm{McF}$ arland turbidity standards $\left(10^{6} \mathrm{cfu} /\right.$ $\mathrm{ml})$. Using a sterilized cotton swab, isolates were inoculated on to Muller Hinton agar. E-strips of vancomycin were applied to it. Incubation was done with controls for $18-24$ hours at $37^{\circ} \mathrm{C}$ aerobically. MIC results were interpreted as susceptible or resistance according to criteria set by CLSI. For vancomycin, MICs $\leq 2 \mu \mathrm{g} / \mathrm{mL}$ were taken into account as sensitive, $4-8 \mu \mathrm{g} / \mathrm{mL}$ intermediate and $\geq 16 \mu \mathrm{g} / \mathrm{mL}$ were considered in resistant zone ${ }^{4,11}$

Statistical analysis for assessment of MIC creep was done by determining different parameters like MIC50, MIC90 (median, 90 $0^{\text {th }}$ percentile), mode, geometrical mean MIC, susceptible and resistant percentages for vancomycin in each year. All the determined susceptibility markers were assessed in each year and plotted over time to assess for vancomycin MIC trends.

Likelihood ratio test was applied to see if the distribution of isolate with $\mathrm{MIC} \geq 1.50 \mu \mathrm{g} / \mathrm{mL}$ for vancomycin differed over the years. $p$-values were reported accurate to three decimal places. 
Vancomycin MIC Creep Phenomenon in MRSA

Table-I: The sources of the clinical methicillin-resistant Staphylococcus aureus (MRSA) isolates (2016-2019).

\begin{tabular}{|c|c|c|c|c|c|c|c|c|c|c|}
\hline \multirow{3}{*}{ Specimen } & \multicolumn{8}{|c|}{ Year } & \multirow{2}{*}{\multicolumn{2}{|c|}{ Total }} \\
\hline & \multicolumn{2}{|c|}{2016} & \multicolumn{2}{|c|}{2017} & \multicolumn{2}{|c|}{2018} & \multicolumn{2}{|c|}{2019} & & \\
\hline & $n$ & $\%$ & $n$ & $\%$ & $n$ & $\%$ & $n$ & $\%$ & $n$ & $\%$ \\
\hline Pus & 43 & 51.2 & 45 & 50.0 & 39 & 45.3 & 48 & 52.2 & 175 & 49.7 \\
\hline Blood & 13 & 15.5 & 15 & 16.7 & 12 & 14.0 & 16 & 17.4 & 56 & 15.9 \\
\hline Wound swab & 11 & 13.1 & 12 & 13.3 & 15 & 17.4 & 13 & 14.1 & 51 & 14.5 \\
\hline Fluids \& Aspirates & 5 & 6.0 & 8 & 8.9 & 6 & 7.0 & 5 & 5.4 & 24 & 6.8 \\
\hline CSF & 4 & 4.8 & 3 & 3.3 & 5 & 5.8 & 3 & 3.3 & 15 & 4.3 \\
\hline Sputum & 3 & 3.6 & 2 & 2.2 & 4 & 4.7 & 2 & 2.2 & 11 & 3.1 \\
\hline CVP tip & 2 & 2.4 & 2 & 2.2 & 3 & 3.5 & 4 & 4.3 & 11 & 3.1 \\
\hline Urine & 3 & 3.6 & 3 & 3.3 & 2 & 2.3 & 1 & 1.1 & 9 & 2.6 \\
\hline Total & 84 & 100.2 & 90 & 100.0 & 86 & 100.0 & 92 & 100.0 & 352 & 100.0 \\
\hline
\end{tabular}

Likelihood ratio $=6.90, \mathrm{P}$-value $=0.998$

\section{RESULTS}

During this study project, over-all 352 MRSA isolates were recovered from 2704 isolates of S. aureus. All MRSA strains were susceptible to vancomycin. MIC for vancomycin remained on rise continuously and by the end of study at year $2019,82.6 \%$ cases were with MIC $\geq 1.5$ as compared to $67.8 \%$ in 2016 . This difference for overall

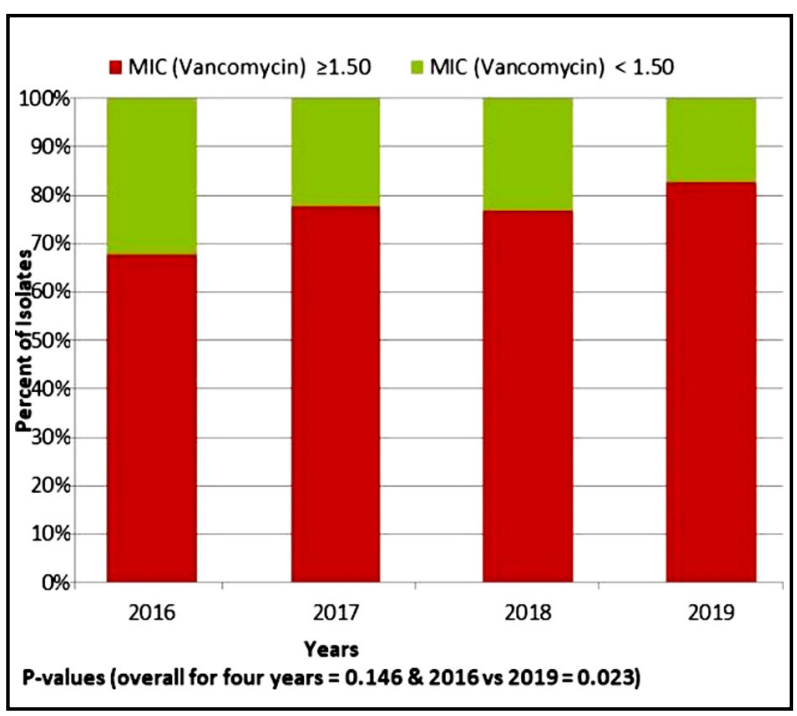

Fig.1: The distribution and trend of vancomycin for clinical isolates of methicillin-resistant Staphylococcus aureus for years 2016-2019. comparison among four years had a p-value 0.146, when compared only between 2016 and 2019 the cases with MIC $\geq 1.5 \mu \mathrm{g} / \mathrm{mL}$ were significantly higher with p-value 0.023. (Fig.1).

Specimen wise distribution of MRSA isolates was observed as well. It was noticed that there was no significant difference for specimen type over the years with $\mathrm{p}$-value $=0.998$. The distribution pattern was not different. Pus, blood and wound

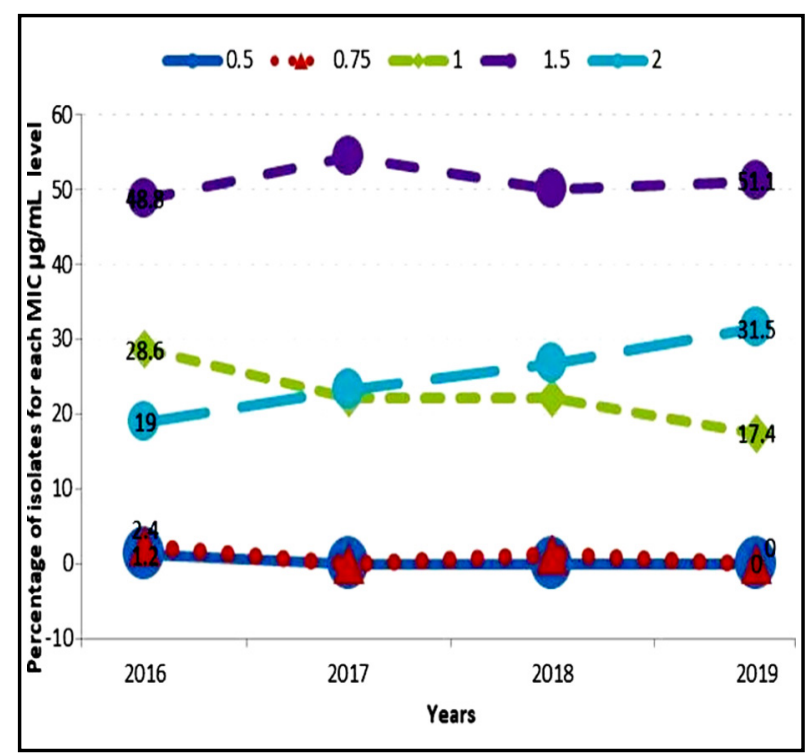

Fig.2: Vancomycin MIC population distribution 2016-2019. 
Faiqa Arshad et al.

Table-II: Statistics and susceptibility regarding vancomycin MICs $(\mu \mathrm{g} / \mathrm{mL})$ for clinical MRSA strains (2016-2019).

\begin{tabular}{|c|c|c|c|c|c|c|c|c|c|c|}
\hline \multirow{2}{*}{ Year } & \multirow{2}{*}{$\begin{array}{l}\text { No of } \\
\text { strains }\end{array}$} & \multirow{2}{*}{$\begin{array}{c}\text { MIC50 } \\
(\mu g / \\
m L)\end{array}$} & \multirow{2}{*}{$\begin{array}{l}\text { MIC90 } \\
(\mu g / m L)\end{array}$} & $\begin{array}{c}M I C<1.5 \\
\mu g / m L\end{array}$ & $\begin{array}{c}M I C \geq 1.5 \\
\mu g / m L\end{array}$ & $\begin{array}{l}\text { Geometric } \\
\text { mean MIC }\end{array}$ & \multirow{2}{*}{$\begin{array}{c}\text { Modal } \\
\text { MIC }\end{array}$} & \multirow{2}{*}{ MIC range } & \multirow{2}{*}{$\begin{array}{l}\text { Percent of } \\
\text { isolates MIC } \\
>\text { baseline } \\
\text { year }{ }^{a} \text { Med- } \\
\text { ian MIC }\end{array}$} & \multirow{2}{*}{$\% S / \% R$} \\
\hline & & & & $n(\%)$ & $n(\%)$ & $\mu g / m L$ & & & & \\
\hline 2016 & 84 & 1.50 & 2.00 & $27(32.1)$ & 57 (67.9) & 1.3 & 1.50 & $(0.5-2.0)$ & -- & $100 / 0$ \\
\hline 2017 & 90 & 1.50 & 2.00 & $20(22.2)$ & $70(77.8)$ & 1.4 & 1.50 & $(1.0-2.0)$ & 9.9 & $100 / 0$ \\
\hline 2018 & 86 & 1.50 & 2.00 & $20(23.3)$ & 66 (76.7) & 1.4 & 1.50 & $(0.75-2.0)$ & 8.8 & $100 / 0$ \\
\hline 2019 & 92 & 1.50 & 2.00 & 16 (17.4) & 76 (82.6) & 1.5 & 1.50 & $(1.0-2.0)$ & 14.7 & $100 / 0$ \\
\hline Total & 352 & 1.50 & 2.00 & $83(23.6)$ & $269(76.4)$ & 1.4 & 1.50 & $(0.5-2.0)$ & 8.5 & $100 / 0$ \\
\hline
\end{tabular}

swab were the three most common specimens for these isolates (Table-I).

For vancomycin, the MIC was also recorded for each case. It was noted that the median MIC, throughout all the years, was $1.5 \mu \mathrm{g} / \mathrm{mL}$ and the $90^{\text {th }}$ percentile was $2.0 \mu \mathrm{g} / \mathrm{mL}$. The geometric mean measured in 2016 was $1.3 \mu \mathrm{g} / \mathrm{mL}$, which increased gradually till 2019 to $1.5 \mu \mathrm{g} / \mathrm{mL}$. In 2016 and 2017 the maximum MIC was $2.0 \mu \mathrm{g} / \mathrm{mL}$ for all years. As compared to 2016, 9.9\% cases increased with MIC greater than 1.5 in 2017. This increment for the year 2018 was $8.8 \%$, and that for 2019 was $14.7 \%$. So in three years, MICs greater than base year median was overall $14.7 \%$ (Table-II).

It can be seen clearly in Fig. 2 that 0.5 and 0.75 MIC was very rare from 2016 to 2019, almost touching zero. The MIC "1.0" had a clear decline from 2016 to 2019, starting from $28.6 \%$ of cases to $17.4 \%$ in 2019 . Conversely, the cases with MIC "2.0" increased during five years from $19.0 \%$ to $31.5 \%$. The maximum number of cases had MIC 1.5 through all the years. These were $48.8 \%$ in 2016, 54.4\% in 2017, 50.0\% in 2018 and then finally $51.1 \%$ in 2019 . A complete shift can be observed between MIC "1.0" and " 2.0 " the percent of cases with MIC " 1.0 " decreased and with MIC "2.0" increased over time crossing each other in 2017.

\section{DISCUSSION}

MRSA has been globally proved to be serious threat to public health. Vancomycin is one of the mainstays for the cure of MRSA infections. However, reduced susceptibility of vancomycin for MRSA infections has been a serious concern over the past few decades. ${ }^{12}$
Our study showed elevated vancomycin MIC for MRSA for four years from 2016-2019 in a large tertiary care institute of Pakistan. Even though there was an absence of VISA and VRSA strains in our study, still a significant shifting trend towards higherMICvaluesraises a seriousconcern regarding potential therapeutic failure by adversely effecting vancomycin activity against MRSA as indicated in a study done in India. ${ }^{13} \mathrm{~A}$ shift towards higher vancomycin MIC values have been documented in studies conducted in different areas worldwide. A similar study conducted in Malaysia discussed that rise in vancomycin MIC value in a time period even being in the susceptible range is labelled as "creep phenomenon". The study mentioned that creep phenomenon cannot be recognized on small scale studies because the difference noted may be very minute and are within susceptible range but has serious clinical impacts in future. To recognize this shift in MIC values, we need a study over a period of few years. Majority of isolates in our study had MIC values $\geq 1.5 \mu \mathrm{g} / \mathrm{mL} .{ }^{14}$ A study done by Cheema et al revealed that $71 \%$ isolates had vancomycin MIC of $2 \mu \mathrm{g} / \mathrm{mL}$ while $29 \%$ isolates had MIC values of $1 \mu \mathrm{g} / \mathrm{mL} .{ }^{15}$ A study conducted by Ejaz et al found that all of the MRSA isolate were sensitive to vancomycin. ${ }^{16}$ However $4 \%$ of the $S$. aureus strains were reported as resistant to vancomycin in a study done in Allied Hospital Faisalabad, Pakistan. ${ }^{17}$ Lower vancomycin MIC values to the strains of MRSA ranged from 0.125 $\mu \mathrm{g} / \mathrm{mL}$ to $1 \mu \mathrm{g} / \mathrm{mL}$ were observed in a study conducted in Nepal. ${ }^{18}$ In our study, 0.5 and 0.75 MIC was very rare throughout 2016 to 2019 as indicated in another study. ${ }^{12}$ In contrast, a study reported that the occurrence of isolates for which the vancomycin MIC was $>1 \mu \mathrm{g} / \mathrm{mL}$ was very 
uncommon, with no increased trend. They tried to find out the possibility of vancomycin MIC creep against MRSA in a large multicenter study conducted in different states of U.S. ${ }^{19}$

Regarding the specimen wise distribution of MRSA isolates, it was found out that pattern was not different by each passing year. Most of the isolates were recovered from samples of pus, blood and wound swabs. Similar findings have been observed in the previous study. ${ }^{20}$ Most of the studies identified the creep phenomenon by using the E test method of susceptibility testing as done in this study..$^{12} \mathrm{E}$ test method is more reliable, sensitive and can accurately detect small MIC changings in vancomycin. ${ }^{8}$

In this study, MIC50, MIC 90 was constant throughout four years period. However, geometric mean MIC raised gradually during the study duration. The MIC greater than base year median was overall $17.3 \%$. Another study reported that geometric mean MIC is a more sensitive marker and reflects the changes in MIC distribution more precisely than traditional susceptibility markers of MIC 50, MIC 90, MIC range and percent susceptibility and percent resistant. ${ }^{21}$ Most studies in Pakistan are not designed to assess more subtle changes in MIC distribution over time. They used traditional markers over a specific period that can disguise tendencies that occur in a given setting over a period of few years. ${ }^{20}$

Studies in different parts of the world have reported vancomycin susceptibility changes over time in single-center and for a similar period of time, as in this study. ${ }^{22,23}$ Yeh et al did study in Taiwan for five years and found an upward trend of vancomycin MIC than baseline year. ${ }^{22}$ Evaluation for creep for three years in a hospital of Portugal reported that creep did not exist in their institution. They suggested that this phenomenon seems not generalized, so each institution should monitor vancomycin MIC values autonomously. ${ }^{6}$ A study was done in two cities of Germany on blood culture isolates by $\mathrm{E}$ test method. They demonstrated that creep exists in City A based on a substantial increase in the number of isolates with a MIC of $1 \mu \mathrm{g} / \mathrm{mL}$ or higher, but there was no change in City B. They also suggested that healthcare settings should observe their own local status of vancomycin MIC values for MRSA strains. ${ }^{8}$

The clinical importance of the increasing trend of vancomycin MIC values, even being in the susceptible range, i.e., creep phenomenon, has been mentioned in various studies. Studies warn that even a minute increase in MIC below the breakpoint is resulting in therapeutic failure when given to patients and is a major medical concern. ${ }^{24}$ A study conducted in Barcelona, Spain, stated that there is a greater probability of therapeutic failure if the strains had a MIC $\geq 1.5 \mu \mathrm{g} / \mathrm{mL}$. Moreover, the death rate related to MRSA bacteremia was significantly higher when vancomycin was empirically used for medication of infectious strains showing high vancomycin MIC values. ${ }^{25}$

Limitations of the study: It is single center study that observed vancomycin MIC creep phenomenon in MRSA isolates of a Tertiary care Hospital of Lahore. We recommend that more studies should be conducted in different hospital of Pakistan to monitor the local Vancomycin MICs Trends over a period of time.

\section{CONCLUSION}

Vancomycin MIC creep was found among clinical MRSA isolates during four years of the study period. Even though there is an absence of VISA and VRSA strains, a significant increase in vancomycin MIC trend within the susceptible range is indeed worrying for the clinicians about the threat of potential therapeutic failure of MRSA infections. Moreover, such studies should be continued in the future in Pakistani hospitals so that we can timely detect the vancomycin-intermediate and resistant strains because the increasing trend of vancomycin can lead the first step to VISA. Moreover, clinical laboratories should implement meticulous techniques for the determination of vancomycin with accurate precision.

\section{Source of Funding: Self.}

Conflict of Interest: None.

\section{REFERENCES}

1. Khan AA, Farooq J, Abid M, Zahra R. Assessment of inducible clindamycin resistance and hyper variable region (HVR) of meca gene in clinical staphylococci. Pak J Med Sci. 2020;36(2):136-140. doi: $10.12669 /$ pjms.36.2.665

2. Islam T, Kubra K, Hassan Chowdhury MM. Prevalence of Methicillin-Resistant Staphylococcus aureus in Hospitals in Chittagong, Bangladesh: A Threat of Nosocomial Infection. J Microsc Ultrastruct. 2018;6(4):188-191. doi: 10.4103/JMAU.JMAU_33_18

3. Hyun I-K, Park PJ, Park D, Choi SB, Han HJ, Song T-J, et al. Methicillin-resistant Staphylococcus aureus screening is important for surgeons. Ann Hepato-Biliary-Pancreatic Surg. 2019;23(3):265-273. doi: 10.14701/ahbps.2019.23.3.265

4. Anitha TK, Rao MR, Shankaregowda R, Mahale RP, Sowmya GS, Chitharagi VB. Evaluation of Vancomycin Minimum Inhibitory Concentration in the clinical isolates of Methicillin Resistant Staphylococcus aureus (MRSA). J Pure Appl Microbiol. 2019;13(3):1797-1801. doi: 10.22207/JPAM.13.3.56 
5. Wilcox M, Al-Obeid S, Gales A, Kozlov R, Martinez-Orozco JA, Rossi F, et al. Reporting elevated vancomycin minimum inhibitory concentration in methicillin-resistant Staphylococcus aureus: Consensus by an International Working Group. Future Microbiol. 2019;14(4):345-352. doi: 10.2217/fmb-2018-0346

6. Joana S, Pedro P, Elsa G, Filomena M. Is vancomycin MIC creep a worldwide phenomenon? Assessment of S. aureus vancomycin MIC in a tertiary university hospital. BMC Res Notes. 2013;6(1):65. doi: 10.1186/1756-0500-6-65

7. Sharma R, Hammerschlag MR. Treatment of MethicillinResistant Staphylococcus aureus (MRSA) Infections in Children a Reappraisal of Vancomycin. Curr Infect Disease Reports. 2019;21(10):37. doi: 10.1007/s11908-019-0695-4

8. Kehrmann J, Kaase M, Szabados F, Gatermann SG, Buer J, Rath PM, et al. Vancomycin MIC creep in MRSA blood culture isolates from Germany: A regional problem? Eur J Clin Microbiol Infect Dis. 2011;30(5):677-683. doi: 10.1007/s10096-010-1140-7

9. Sneha S, Venishetty S, Seshadri S, Sudhakar Rao M, Mukhopadhyay C. An unusual occurrence of methicillin resistant staphylococcal endocarditis with vancomycin creep phenomenon - A therapeutic chall. J Clin Diagnostic Res. 2016;10(12):OD12-OD14. doi: 10.7860/JCDR/2016/21709.9085

10. Chen CP, Liu MF, Lin CF, Lin SP, Shi ZY. The association of molecular typing, vancomycin MIC, and clinical outcome for patients with methicillin-resistant Staphylococcus aureus infections. J Microbiol Immunol Infect. 2017;50(5):619-626. doi: 10.1016/j.jmii.2015.08.015

11. Clinical and Laboratory Standard Institute (CLSI). Performance Standards for Antimicrobial Susceptibility Testing. CLSI supplement M100. 29th ed. Wayne, PA: Clinical and Laboratory Standard Institute; 2019.

12. Chang CN, Lo WT, Chan MC, Yu CM, Wang CC. An investigation of vancomycin minimum inhibitory concentration creep among methicillin-resistant Staphylococcus aureus strains isolated from pediatric patients and healthy children in Northern Taiwan. J Microbiol Immunol Infect. 2017;50(3):362369. doi: 10.1016/j.jmii.2015.05.013

13. Dhawan B, Gadepalli R, Rao C, Kapil A, Sreenivas V. Decreased susceptibility to vancomycin in meticillin-resistant Staphylococcus aureus: A 5 year study in an Indian tertiary hospital. J Med Microbiol. 2010;59(3):375-376. doi: 10.1099/ jmm.0.011940-0

14. Ahmad N, NawiS, Rajasekaran G, Maning N, Aziz MN, Husin A, et al. Increased vancomycin minimum inhibitory concentration among Staphylococcus aureus isolates in Malaysia. J Med Microbiol. 2010;59(12):1530-1532. doi: 10.1099/jmm.0.022079-0

15. Cheema KH, Javed I, Mushtaq S. Anwar MS. Heteroresistant Vancomycin Intermediate Staphylococcus aureus in a tertiary care hosptital. Biomedica. 2017;33(3):192-196.

16 Ejaz H. Determination of bacterial profile and spectrum of antimicrobial drug resistance in pediatric wound infections. J Pure Appl Microbiol. 2019;13(4):2097-2104. doi: 10.22207/ JPAM.13.4.21

17. Junaid K, Mustafa AUL, Arshad S, Farraj DAAL, Younas S, Ejaz H. Burn wound infections: A serious threat of multidrugresistant Staphylococcus aureus. Pak J Med Heal Sci. 2019;13(3):804-807.

18. Kshetry AO, Pant ND, Bhandari R, Khatri S, Shrestha KL, Upadhaya SK, et al. Minimum inhibitory concentration of vancomycin to methicillin resistant Staphylococcus aureus isolated from different clinical samples at a tertiary care hospital in Nepal. Antimicrob Resist Infect Control. 2016;5(1):4-9. doi: 10.1186/s13756-016-0126-3
19 Sader HS, Fey PD, Fish DN, Limaye AP, Pankey G, Rahal J, et al. Evaluation of vancomycin and daptomycin potency trends (MIC creep) against methicillin-resistant Staphylococcus aureus isolates collected in nine U.S. Medical Centers from 2002 to 2006. Antimicrob Agents Chemother. 2009;53(10):4127-4132. doi: 10.1128/AAC.00616-09

20. Kaleem F. Current status of vancomycin susceptibility against methicillin resistant Staphylococcus aureus (MRSA) strains: A study at two tertiary care hospitals of Pakistan. African J Microbiol Res. 2012;6(33):6243-6246. doi: 10.5897/ AJMR11.787

21. Steinkraus G, White R, Friedrich L. Vancomycin MIC creep in non-vancomycin-intermediate Staphylococcus aureus (VISA), vancomycin-susceptible clinical methicillin-resistant $S$. aureus (MRSA) blood isolates from 2001-05. J Antimicrob Chemother. 2007;60(4):788-794. doi: 10.1093/jac/dkm258

22 Yeh YC, Yeh KM, Lin TY, Chiu SK, Yang YS, Wang YC, et al. Impact of vancomycin MIC creep on patients with methicillin-resistant Staphylococcus aureus bacteremia. J Microbiol Immunol Infect. 2012;45(3):214-220. doi: 10.1016/j. jmii.2011.11.006

23. Wang G, Hindler JF, Ward KW, Bruckner DA. Increased vancomycin MICs for Staphylococcus aureus clinical isolates from a university hospital during a 5-year period. J Clin Microbiol. 2006;44(11):3883-3886. doi: 10.1128/JCM.01388-06

24. Sakoulas G, Moise-Broder PA, Schentag J, Forrest A, Moellering RC, Eliopoulos GM. Relationship of MIC and bactericidal activity to efficacy of vancomycin for treatment of methicillinresistant Staphylococcus aureus bacteremia. J Clin Microbiol. 2004;42(6):2398-2402. doi: 10.1128/JCM.42.6.2398-2402.2004

25. Soriano A, Marco F, Martinez J, Pisos E, Almela M, Dimova VP, et al. Influence of vancomycin minimum inhibitory concentration on the treatment of methicillin-resistant Staphylococcus aureus bacteremia. Clin Infect Dis. 2008;46(2):193-200. doi: $10.1086 / 524667$

\section{Authors' Contribution:}

FA: Entire Research work (conception \& design, Data analysis\& interpretation). Responsible and accountable for the accuracy or integrity of the work.

SS: Conceptualization \& Overall supervision of research work, Final approval of version to be submitted. Responsible and accountable for the accuracy or integrity of the work.

SJ, RT: Supervision of research work, Revising critically for important intellectual content.

Authors:

1. Dr. Faiqa Arshad Ph.D. Scholar. Department of Microbiology

2. Dr. Sidrah Saleem, MBBS, M.Phil., PhD (Microbiology), Professor \& Head, Department of Microbiology,

3. Dr. Shah Jahan, PhD (Molecular Biology), Associate Professor,

Department of Immunology,

4. Dr. Romeeza Tahir, M.Sc., M.Phil., PhD (Immunology) Assistant Professor, Department of Immunology,

1-4: University of Health Sciences, Lahore, Pakistan. 